\title{
A large community outbreak of Legionnaires' disease in Vila Franca de Xira, Portugal, October to November 2014
}

T Shivaji (tarashivaji@dgs.pt) ${ }^{1,2}$, C Sousa Pinto ${ }^{1}$, A San-Bento ${ }^{1}$, L A Oliveira Serra ${ }^{1}$, J Valente ${ }^{1}$, J Machado ${ }^{3}$, T Marques $^{1}$, L

Carvalho $^{4}$, P J Nogueira ${ }^{1}$, B Nunes ${ }^{3}$, P Vasconcelos ${ }^{1}$

1. Directorate General of Health, Lisbon, Portugal

2. European Intervention Epidemiology Training Programme (EPIET), European Centre for Disease Prevention and Control (ECDC), Stockholm, Sweden

3. National Institute of Health Dr Ricardo Jorge, Lisbon, Portugal

4. Centro de Estatística e Aplicações Universidade de Lisboa (Centre for Statistics and Applications of the University of Lisbon), Lisbon, Portugal

An outbreak of Legionnaires' disease with 334 confirmed cases was identified on 7 November 2014 in Vila Franca de Xira, Portugal and declared controlled by 21 November. Epidemiological, environmental and microbiological analysis identified industrial wet cooling systems to be the probable source of infection. Preliminary results from sequence-based typing of clinical specimens and environmental isolates confirmed this link. A series of meteorological phenomena are likely to have contributed to the scale of this outbreak.

On Friday 7 November, the Portuguese Directorate General of Health (DGS) was informed of 18 cases of Legionnaires' disease admitted to two hospitals within 24 hours in the municipality of Vila Franca de Xira, Lisbon. No cases reported foreign travel and all had positive urinary antigen tests for Legionella pneumophila serogroup 1. Between 2007 and 2012, the annual number of cases reported in Portugal had ranged from 82 to $140[1,2]$. Within hours, the Ministries of Health and Environment convened a multidisciplinary task force to investigate and control the outbreak.

\section{Description of the investigation}

\section{Epidemiological investigation}

Cases of Legionnaires' disease and/or laboratory diagnoses of Legionella are mandatorily notifiable in Portugal. All notifications since 1 October 2014 were reviewed. The DGS and the national reference laboratory (NRL) actively searched for cases through hospital and laboratory networks.

A confirmed case was defined as a person with pneumonia, laboratory confirmation of L. pneumophila, symptom onset after 1 October and a history of living in, working in, or visiting Vila Franca de Xira from 15 September onwards. All reported cases with clinical symptoms of pneumonia and an epidemiological connection to the affected area were treated as suspected cases and investigated further.

Staff from regional health authorities interviewed patients applying a standard questionnaire for symptoms, risk factors, place of residence and work, recent travel and daily habits during the incubation period. The outbreak was described in terms of date of symptom onset, age and sex distributions. Street addresses were geocoded using Google Earth to determine spatial distribution.

Back-calculation models developed by Egan et al. [3] were applied using epidemiological data available on 12 November. The models allowed determination of the period of aerosol release (period of exposure) and estimation of the number of expected cases. At the early phase of the outbreak, we had limited information on exposure. It was not clear which case represented the index case, therefore two scenarios were considered based on the available date of symptom onset: 14 October and 21 October.

\section{Environmental investigation}

As the cooling towers of wet cooling systems are often implicated in large outbreaks of Legionnaires' disease, the municipality identified all premises with wet cooling systems and other aerosol-generating sources in Vila Franca de Xira. Water samples were taken from public, domestic and industrial sources. The locations of wet cooling systems were georeferenced using visual pinpointing in Google Earth. Meteorological and 
Confirmed cases of Legionnaires' disease by date of symptom onset, Vila Franca de Xira, Portugal, 14 October - 24 November $2014(\mathrm{n}=334)$

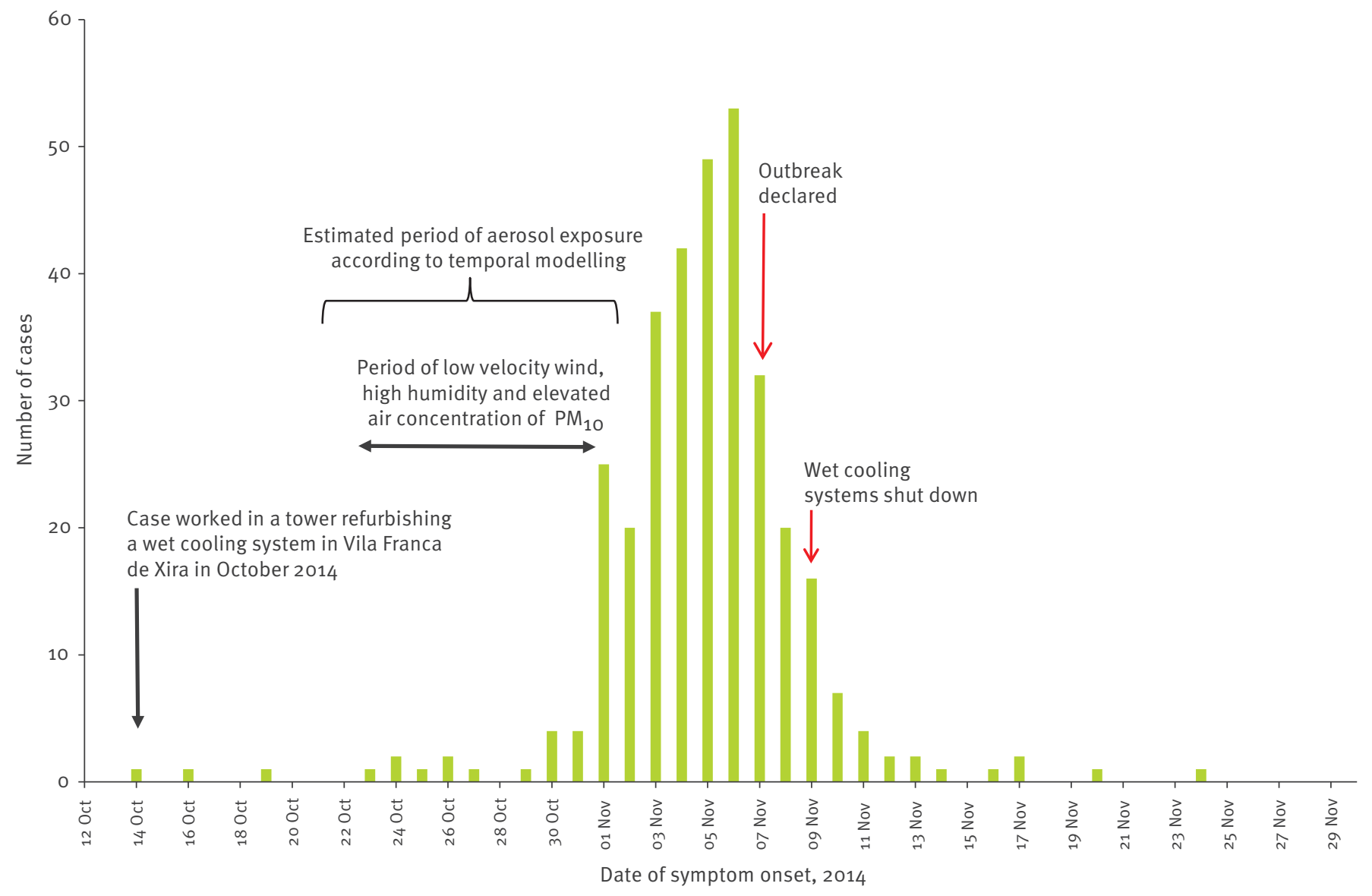

environmental agencies analysed weather data from 1 October until 7 November.

\section{Microbiological investigation}

The NRL tested clinical specimens and environmental samples for Legionella species. They performed sequence-based typing (SBT) following the protocol of the European Working Group for Legionella infections [4] with the aim of matching environmental isolates with isolates from clinical specimens.

\section{Measures taken}

On 7 November, hospitals in the region of Lisbon and the Tagus Valley activated the contingency plan to respond to the increased demand for ventilator systems. As the investigations were ongoing, the following actions were taken in the municipality: on 8 November, the public water authority raised chlorine levels and public pools, spas and fountains were shut down and disinfected after sampling; on 9 November, the Environmental Inspectorate shut down and ordered the disinfection of all wet cooling systems operating in the municipality.

\section{Outbreak description}

By 11 December 2014, the DGS had received reports of 417 suspected cases of Legionnaires' disease of which 334 were confirmed. All 334 had positive urinary antigen tests for $L$. pneumophila serogroup 1 . Of the confirmed cases, 221 (67\%) were male, the median age was 58 years (range: 25-92 years). Ten deaths were reported in confirmed cases and a further two deaths were awaiting post mortem investigations.

The outbreak started on 14 October, with a sharp increase in cases from 1 November, and peaked on 6 November (Figure1). The last reported case developed symptoms on 24 November. No confirmed cases with symptom onset after 24 November have been notified. The outbreak was considered controlled on 21 November. By 11 December, two incubation periods had passed since the peak of the outbreak and the implementation of control measures, and the number of notified cases from the area had returned to levels observed before the outbreak.

Place of residence was mapped for 250 of the 294 cases notified by 14 November, and the geospatial distribution was analysed: $90 \%(n=226)$ lived within $3 \mathrm{~km}$ of a wet cooling system. The task force hypothesised 


\section{FIGURE 2}

Mapping of cases of Legionnaires' disease by place of residence, Vila Franca de Xira, Portugal, notified by 14 November $2014(\mathrm{n}=250)$

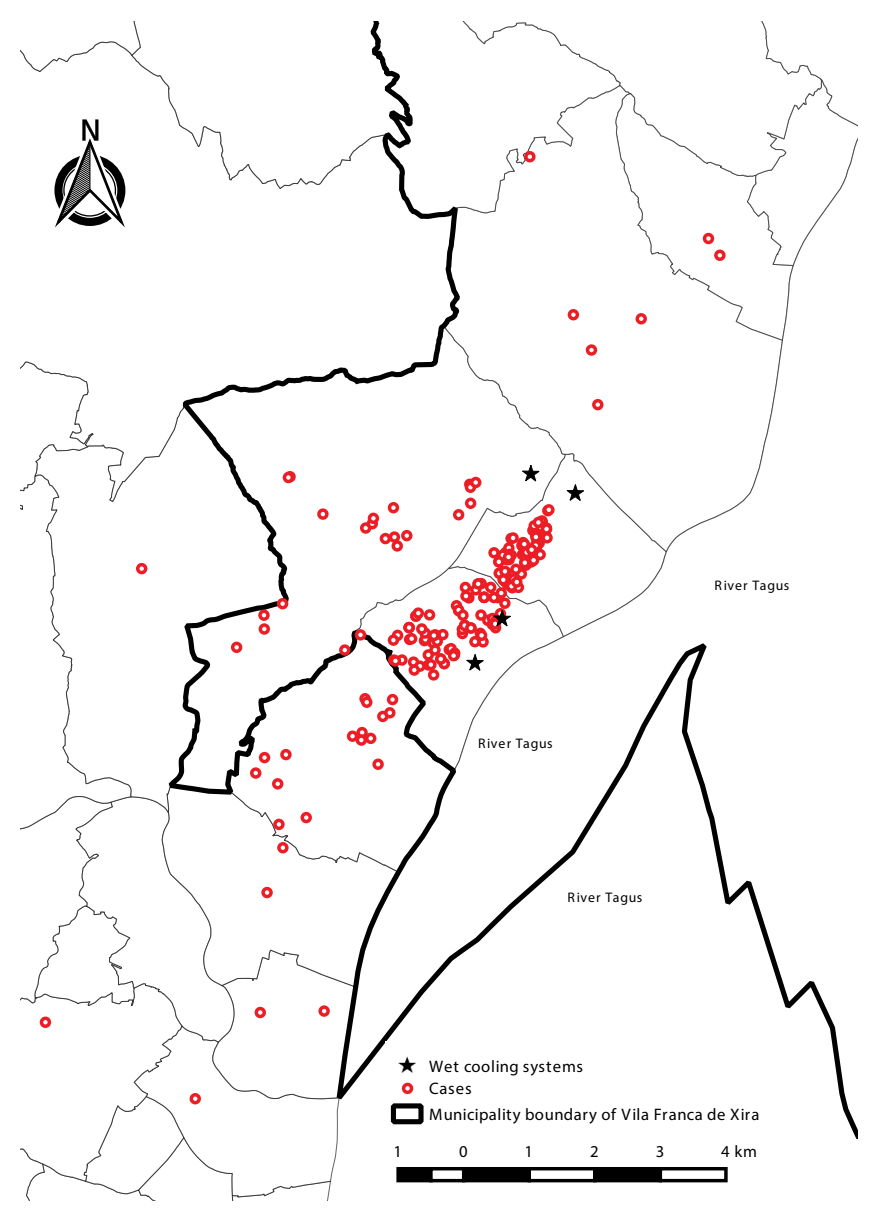

that the most likely source of infection was aerosolised release from one or more of these systems (Figure 2).

The back-calculation models using 21 October as the date of first symptom onset, suggested a logistic growth of the environmental exposure, with greatest intensity most probably between 23 October and 2 November. From this analysis, we estimated a total outbreak size of 325 cases ( $95 \% \mathrm{Cl}: 287-422)$. Since cases had been identified with symptom onset as early as 14 October, detailed interviews were conducted with them to ascertain if they were indeed linked to this outbreak. The patient who developed symptoms on 14 October was found to have been involved in maintenance works in the towers of local industrial wet cooling systems in the two weeks before onset of symptoms.

Meteorological data from the weather station in Alverca, $8 \mathrm{~km}$ from Vila Franca de Xira, indicated north-easterly wind with a velocity of $2-3 \mathrm{~m} / \mathrm{s}$ between 18 October and 1 November. This wind coincided with high humidity (80\%) from 26 October onwards, particularly at night. In addition, between 19 October and 1 November, a cloud of sand and dust lifted by a storm in the Sahara desert, covered Portugal and increased airborne concentrations of small particulate matter $\left(P M_{10}\right)$.

By 20 November, 129 respiratory secretion specimens and 101 environmental samples were under analysis. Preliminary results from 12 clinical specimens identified the causative organism as L. pneumophila serogroup 1, sequence type 11, 14, 16, 10, 15, 13, 2ST 1905. Preliminary results of an environmental sample from once cooling system also showed sequence type 11, 14, $16,10,15,13,2$ ST 1905.

\section{Discussion}

This was the largest outbreak of Legionnaires' disease in Portugal to date and among the largest reported in Europe. In the largest outbreak with 449 confirmed cases in Murcia, Spain, in 2001, a microbiological link between cases and a hospital cooling tower was demonstrated in the months after the outbreak [5]. Timely collection of respiratory secretion specimens by hospitals and widespread environmental sampling in the early phase of the outbreak allowed identification of a microbiological link between the environment samples and patient isolates [6].

To the best of our knowledge, this is the first time the models of Egan et al. [3] have been used in the early stage of an outbreak investigation. At the time of applying the models, we strongly suspected cooling towers as a source but had not ruled out other sources. Using the models we were able to identify cases whose pattern of illness did not fit with an aerosolised exposure. The occupational exposure of these outliers was identified within one week of the declaration of the outbreak.

Microbiological results suggested a link between the isolate from a wet cooling system and the isolates from the clinical specimens of patients, consistent with previously described large community outbreaks in Spain and the United Kingdom [5,7].

The concordance between the independent analysis of meteorological conditions and temporal modelling supports the theory that the prevailing weather conditions created a unique setting for Legionella multiplication and may explain the large scale of the outbreak. Unseasonable weather [8] and periods of humidity [9] have previously been described as facilitating the circulation of aerosolised Legionella.

\section{Conclusion}

Although the outbreak is considered controlled, detailed epidemiological investigations and microbiological investigations continue and may result in the reclassification of cases and an increase in confirmed cases. In addition, determination of the monoclonal subtype and molecular sequence typing to provide further evidence about the link between the environment and patients are underway. An evaluation of the outbreak with the view to improving early detection will be developed. . 


\section{Acknowledgements}

The authors would like to acknowledge: Dr Francisco George - Director General of Health Portugal; Fernando Almeida President of the National Institute of Health Dr Ricardo Jorge; Miguel Miranda - President of the Portuguese Meteorological Institute (IPMA); Nuno Lacasta - President of the Portuguese Environmental Agency; Cristina Abreu dos Santos of the Directorate General of Health; Isabel Falcão of the Directorate General of Health; Marina de Lurdes Pires Ramos do Nascimento, Paulo Feliciano Diegues of the Directorate General of Health; Ricardo Mexia of the National Institute of Health Dr Ricardo Jorge; Luis Cadinha, Eugénio Cordeiro, Ana Maria Correia of the Regional Health Administration of Central Portugal; Members of the task force; The primary, secondary and critical care services of the regional health administrations in Portugal.

\section{Conflict of interest}

None declared.

\section{Authors' contributions}

Tara Shivaji wrote the manuscript. Lucilia Carvalho modelled the outbreak and provided insight into interpretation of results. Jorge Machado provided details on microbiological investigation and results. Teresa Marques provided technical details and insight on microbiological investigation and interpretation of results. Paulo Jorge Nogueira: modelled the outbreak. Baltazar Nunes modelled the outbreak and interpreted results for the manuscript. Catia Sousa Pinto provided technical details on epidemiological investigations and results Ana San-Bento was involved in epidemiological analysis for the manuscript. Luis Antonio Oliveira Serra georeferenced data and produced the map and technical commentary. João Valente was involved in epidemiological analysis and provided technical commentary on the manuscript. Paula Vasconcelos provided technical information and edited the manuscript.

\section{References}

1. European Centre for Disease Prevention and Control (ECDC). Legionnaires' disease in Europe, 2012. Stockholm: ECDC; 2014. Available from: http://www.ecdc.europa.eu/en/publications/ Publications/legionnaires-disease-surveillance-2012.pdf

2. European Centre for Disease Prevention and Control (ECDC). Annual Epidemiological report 2013. Reporting on 2011 surveillance data and 2012 epidemic intelligence data. Stockholm: ECDC; 2013 . Available from: http://www. ecdc.europa.eu/en/publications/Publications/AnnualEpidemiological-Report-2013.pdf

3. Egan JR, Hall IM, Lemon DJ, Leach S. Modeling Legionnaires' disease outbreaks: estimating the timing of an aerosolized release using symptom-onset dates. Epidemiology.

2011;22(2):188-98. Available from: http://dx.doi.org/10.1097/ EDE.obo13e31820937c6 PMID:21242803

4. Gaia V, Fry NK, Afshar B, Lück PC, Meugnier H, Etienne J, et al. Consensus sequence-based scheme for epidemiological typing of clinical and environmental isolates of Legionella pneumophila. J Clin Microbiol. 2005;43(5):2047-52. Available from: http://dx.doi.org/10.1128/JCM.43.5.2047-2052.2005 PMID:15872220

5. García-Fulgueiras A, Navarro C, Fenoll D, García J, GonzálezDiego P, Jiménez-Buñuales T, et al. Legionnaires' disease outbreak in Murcia, Spain. Emerg Infect Dis. 2003;9(8):915-21. Available from: http://dx.doi.org/10.3201/eido908.030337 PMID:12967487

6. Chasqueira M, Rodrigues L, Nascimento M, Ramos M, Marques T. Genetic diversity and evolutionary relationships among Legionella pneumophila clinical isolates, Portugal, 1987 to 2012. Euro Surveill. 2014;19(46):20965. Available from
http://dx.doi.org/10.2807/1560-7917.ES2014.19.46.20965 PMID:25425515

7. Keramarou M, Evans MR, South Wales Legionnaires Disease Outbreak Control Team. A community outbreak of Legionnaires' disease in South Wales, August-September 2010. Euro Surveill. 2010;15(42):pii19691. Available from: PMID:21034723

8. Dunn CE, Rowlingson B, Bhopal RS, Diggle P. Meteorological conditions and incidence of Legionnaires' disease in Glasgow, Scotland: application of statistical modelling. Epidemiol Infect. 2013;141(4):687-96. Available from: http://dx.doi.org/10.1017/ So95026881200101X PMID:22687530

9. Nygård K, Werner-Johansen $\emptyset$, Rønsen S, Caugant DA, Simonsen $\varnothing$, Kanestrøm A, et al. An outbreak of legionnaires disease caused by long-distance spread from an industrial air scrubber in Sarpsborg, Norway. Clin Infect Dis. 2008;46(1):619. Available from: http://dx.doi.org/10.1086/524016 PMID:18171215 